\title{
BUMPY METRICS ON SPHERES AND MINIMAL INDEX GROWTH
}

\author{
HANS-BERT RADEMACHER \\ Dedicated to Paul Rabinowitz with best wishes
}

\begin{abstract}
The existence of two geometrically distinct closed geodesics on an $n$-dimensional sphere $S^{n}$ with a non-reversible and bumpy Finsler metric was shown independently by Duan \& Long [7] and the author [25. We simplify the proof of this statement by the following observation: If for some $N \in \mathbb{N}$ all closed geodesics of index $\leq N$ of a non-reversible and bumpy Finsler metric on $S^{n}$ are geometrically equivalent to the closed geodesic $c$ then there is a covering $c^{r}$ of minimal index growth, i.e.$$
\operatorname{ind}\left(c^{r m}\right)=m \operatorname{ind}\left(c^{r}\right)-(m-1)(n-1)
$$

for all $m \geq 1$ with ind $\left(c^{r m}\right) \leq N$. But this leads to a contradiction for $N=\infty$ as pointed out by Goresky \& Hingston [13]. We also discuss perturbations of Katok metrics on spheres of even dimension carrying only finitely many closed geodesics. For arbitrarily large $L>0$ we obtain on $S^{2}$ a metric of positive flag curvature carrying only two closed geodesics of length $<L$ which do not intersect.
\end{abstract}

\section{INTRODUCTION}

In this note we consider existence results for closed geodesics on spheres $S^{n}$ of dimension $n$ endowed with a Finsler metric $f$. The Finsler metric $f$ is called reversible, if $f(-X)=f(X)$ holds for all tangent vectors $X$. Otherwise we call the metric non-reversible. Note that two closed geodesics $c_{1}, c_{2}: S^{1} \longrightarrow M$ of a non-reversible Finsler metric are geometrically equivalent if their images $c_{1}\left(S^{1}\right)=c_{2}\left(S^{1}\right)$ and their orientations coincide. In the reversible case $c_{1}, c_{2}$ are geometrically equivalent if and only if $c_{1}\left(S^{1}\right)=c_{2}\left(S^{1}\right)$. Closed geodesics which are not geometrically equivalent are called geometrically distinct. We call a closed geodesic prime if it is not the covering of a shorter closed curve. For $m \geq 1$ and a prime closed geodesic $c$ of a non-reversible Finsler metric the coverings $c^{m}$ defined by $c^{m}(t)=c(m t)$ are geometrically equivalent closed geodesics.

A closed geodesic $c: S^{1} \longrightarrow S^{n}$ is non-degenerate if its nullity vanishes. The nullity is the dimension of the kernel of the index form minus one, it

Date: 2016-08-04, revised: 2016-09-02.

2010 Mathematics Subject Classification. 53C22, 58E10.

Key words and phrases. closed geodesic, free loop space, minimal index growth, Morse inequalities, Katok metric, positive flag curvature. 
equals the dimension of periodic Jacobi fields orthogonal to the velocity field $c^{\prime}$ of the closed geodesic.

A Finsler metric $f$ on a compact manifold $M$ is called bumpy if all closed geodesics are non-degenerate. In this case the energy functional $E: \Lambda S^{n} \longrightarrow$ $\mathbb{R}$ defined on the free loop space $\Lambda S^{n}$ carrying a canonical $S^{1}$-action can be viewed as a Morse function whose critical set decomposes into a disjoint union of non-degenerate $S^{1}$-orbits of closed geodesics. Following ideas by Birkhoff [4, Sec.17], [5, p.135-139] the existence of a single closed geodesic on a compact and simply-connected manifold with a Finsler metric was shown by Lusternik \& Fet [21]. It turns out that existence results for several closed geodesics strongly depend on the reversibility of the metric. The sequence (ind $\left.\left(c^{m}\right)\right)_{m>1}$ of Morse indices of the coverings $c^{m}$ of a closed geodesic $c$ plays an important role in existence proofs for several closed geodesics. It is a result by Fet [12] that a bumpy and reversible Finsler metric on a compact and simply-connected manifold carries at least two geometrically distinct closed geodesics. An analogous statement holds also for non-reversible Finsler metrics on spheres as shown independently by Duan-Long in [7] and the author in [25]:

Theorem 1. 7], 25] A bumpy and non-reversible Finsler metric on a sphere $S^{n}$ of dimension $n \geq 3$ carries two geometrically distinct closed geodesics.

In [7] the classification of the symplectic normal forms of the linearized Poincaré mapping of a closed geodesic and a case distinction is used. In [25] the common index jump theorem due to [20, Thm.4.3] is the main ingredient. The statement of Theorem 1 as well as the statement of the following Proposition 1 also hold for compact and simply-connected manifolds $M$ which are rationally homotopy equivalent to an $n$-sphere $S^{n}$. In this note we show that one can use the following result about the index growth, i.e. the growth of the sequence (ind $\left.\left(c^{m}\right)\right)_{m \geq 1}$ which also gives a short proof of Theorem 1 ;

Proposition 1. Let $f$ be a bumpy and non-reversible Finsler metric on $S^{n}$ and let $N_{1} \geq 5 n$ be a number such that the following assumption holds: There is a prime closed geodesic $c$ such that all closed geodesics d whose index satisfies $\operatorname{ind}(d) \leq N_{1}+2$ are geometrically equivalent to $c$. Let $r=n$ if $n$ is even and $r=(n+1) / 2$ if $n$ is odd. Let $m_{1}:=\max \left\{m \in \mathbb{N}\right.$; $\left.\operatorname{ind}\left(c^{r m}\right) \leq N_{1}\right\}$. Then $m_{1} \geq 2$ and the closed geodesic $c^{r}$ is of minimal index growth up to level $m_{1}$, i.e.

$$
\operatorname{ind}\left(c^{r m}\right)=m \operatorname{ind}\left(c^{r}\right)-(m-1)(n-1)
$$

holds for all $m \leq m_{1}$. The closed geodesic $c$ is of elliptic-parabolic type, i.e. its linearized Poincaré mapping decomposes into rotations.

We prove this Proposition in the next section. In the case $n=2, N_{1}=\infty$ it was shown by Ziller in [26, p.149] that Equation (1) holds with $r=2$ and leads to a contradiction.

The closed geodesic $c^{r}$ satisfying Equality (11) is called of minimal index growth up to level $m_{1}$. This is motivated by the following Inequality (2). On 
the other hand the following Inequality (3) shows that Equation (11) can hold only for finitely many $m$ which shows that Proposition 1 implies Theorem 1 for $N_{1}=\infty$.

Proposition 2. (cf. Goresky \& Hingston [13, Prop.6.1]) Let c be a closed geodesic of a bumpy Finsler metric. Then for all $m \geq 1$ :

$$
\operatorname{ind}\left(c^{r m}\right) \geq m \operatorname{ind}\left(c^{r}\right)-(m-1)(n-1) .
$$

And there is an $N \in \mathbb{N}$ such that for all $m>N$ :

$$
\operatorname{ind}\left(c^{r m}\right) \geq m \operatorname{ind}\left(c^{r}\right)-(m-1)(n-1)+1 .
$$

This Proposition is a particular case of [13, Prop.6.1]. It is a direct consequence of Bott's formula for ind $\left(c^{m}\right)$, cf. [6, Thm.A]. The statement of the Proposition can also be shown without the assumption that the metric is bumpy.

It is a remarkable result by Bangert \& Long [3] that for any non-reversible Finsler metric on $S^{2}$ there are two closed geodesics. There are a number of results for metrics in higher dimensions with and without non-degeneracy assumptions or with curvature assumptions, cf. for example [8], [9], [10], [11], [17] and [24].

There are non-reversible bumpy Finsler metrics of constant flag curvature depending on an irrational parameter on $S^{2 n-1}$ resp. $S^{2 n}$ with exactly $2 n$ geometrically distinct closed geodesics. On the 2-sphere for any $N_{1} \in \mathbb{N}$ there is a Katok metric satisfying the assumptions of Proposition 1. These metrics occured in a paper by Katok [18, their geometry is investigated by Ziller in [26]. By construction these metrics are invariant under a ro-

tation, the closed geodesics $c_{1}, c_{1}^{-1}, \ldots, c_{n}, c_{n}^{-1}$ occur in pairs differing by orientation and length. They are fixed point sets of reflections at a twodimensional plane. In Section 3 we show that for a given arbitrarily large $L$ one can perturb these metrics on $S^{2 n}$ to obtain a non-reversible Finsler metric with positive flag curvature carrying exactly $2 n$ geometrically distinct closed geodesics $d_{1}, d_{2}, \ldots, d_{2 n}$ with length $<L$, which pairwise do not intersect. Hence for these closed geodesics the closed curves $d_{j}^{-1}, j=1,2, \ldots, 2 n$ with opposite orientation are not geodesics. These metrics are also invariant under a rotation and are obtained by breaking the symmetry with respect to the reflections at two-dimensional planes.

In dimension 2 this produces for $k \geq 4$ in any $C^{k}$-neighborhood of the Katok metric examples of non-reversible Finsler metrics on $S^{2}$ of positive flag curvature with two simple closed geodesics, which do not intersect.

\section{ACKNOWLEDGEMENT}

I am grateful to Nancy Hingston for helpful discussions about the topic of the paper and her comments on an earlier version. And I want to thank Philip Kupper for his careful reading and the referee for his suggestions. 


\section{Proof of Proposition 1}

We denote by $f: T S^{n} \longrightarrow \mathbb{R}$ the Finsler metric defined on the tangent bundle $T S^{n}$ of the $n$-sphere. We consider the free loop space $\Lambda S^{n}$ of $H^{1}$ maps $\sigma: S^{1}=[0,1] /\{0,1\} \longrightarrow S^{n}$ on the sphere $S^{n}$.

Closed geodesics are the critical points of the energy functional

$$
E: \Lambda S^{n} \longrightarrow \mathbb{R} ; E(\sigma)=\frac{1}{2} \int_{0}^{1} f^{2}\left(\sigma^{\prime}(t)\right) d t .
$$

There is a canonical $S^{1}$-action $(z, \sigma) \in S^{1} \times \Lambda S^{n} \mapsto z . c \in \Lambda S^{n}$ defined by $z . c(t)=c(t+z)$ on the free loop space leaving the energy functional invariant. The index form $\mathcal{H}_{c}$ of the closed geodesic can be identified with the hessian $d^{2} E(c)$ of the energy functional at $c$ by the second variational formula. If the metric is bumpy one can view the energy functional as a Morse function on the quotient space $\Lambda S^{n} / S^{1}$, for details see for example [25, Sec.2].

We assume that there is a prime closed geodesic $c$ such that any other closed geodesic $d$ with $\operatorname{ind}(d) \leq N_{1}+2$ satisfies $d=z \cdot c^{m}$ for some $z \in S^{1}$ and $m \geq 1$.

Let $\Lambda^{0} S^{n}=\left\{\sigma \in \Lambda S^{n} ; E(\sigma)=0\right\}$ be the set of point curves, which one can identify with the sphere $S^{n}$. We denote by $b_{k}:=b_{k}\left(\Lambda S^{n} / S^{1}, \Lambda^{0} S^{n} / S^{1} ; \mathbb{Q}\right)$ the Betti numbers of the quotient space pair $\left(\Lambda S^{n} / S^{1}, \Lambda^{0} S^{n} / S^{1}\right)$ with rational coefficients.

Let $\Lambda(c):=\left\{\sigma \in \Lambda S^{n} ; E(\sigma)<E(c)\right.$. Then the Betti numbers of the local homology produced by the covering $c^{m}$ are given by:

$$
\begin{aligned}
b_{j}\left(c^{m}\right) & =\operatorname{dim} H_{j}\left(\left(\Lambda\left(c^{m}\right) \cup S^{1} \cdot c^{m}\right) / S^{1}, \Lambda\left(c^{m}\right) / S^{1} ; \mathbb{Q}\right) \\
& = \begin{cases}1 \quad & j=\operatorname{ind}\left(c^{m}\right) \text { and } \\
0 \quad & \text { otherwise } \operatorname{ind}\left(c^{2}\right) \equiv \operatorname{ind}(c) \quad(\bmod 2) .\end{cases}
\end{aligned}
$$

For $k \leq N_{1}+2$ :

(4) $w_{k}=\#\left\{m \in \mathbb{N} ; \operatorname{ind}\left(c^{m}\right)=k, m \equiv 1\right.$ or $\left.\operatorname{ind}\left(c^{2}\right) \equiv \operatorname{ind}(c) \quad(\bmod 2)\right\}$

gives the number of critical points of index $k$ of the Morse function $E$ : $\Lambda S^{n} / S^{1} \longrightarrow \mathbb{R}$ producing non-trivial local homology.

Bott's formula for the indices ind $\left(c^{m}\right)$ of iterates $c^{m}$ implies the following statements about the parity of ind $\left(c^{m}\right), m \geq 1$ :

$$
\operatorname{ind}\left(c^{2 m+1}\right) \equiv \operatorname{ind}(c) ; \operatorname{ind}\left(c^{2 m}\right) \equiv \operatorname{ind}\left(c^{2}\right) \quad(\bmod 2),
$$

cf. [22, Sec.1]. This observation implies that $w_{k}=0$ for all numbers $k \equiv$ $\operatorname{ind}(c)+1(\bmod 2)$ for all $k \leq N_{1}+2$. Hence the equality case holds in the Morse inequalities:

$$
w_{k}=b_{k}, k \leq N_{1},
$$

cf. [22, (2.3)]. Since Bott's formula also implies ind $\left(c^{m}\right) \geq \operatorname{ind}(c) ; m \geq 1$ we conclude

$$
\operatorname{ind}(c)=n-1 \text {. }
$$


Here we use that $b_{0}=b_{1}=\ldots=b_{n-2}=0, b_{n-1}=1$, cf. [22, Thm.2.4] resp. [15, p.104]. Equation (7) implies that the sequence $\left(\operatorname{ind}\left(c^{m}\right)\right)_{m \geq 1}$ is monotone increasing, i.e.

$$
\operatorname{ind}\left(c^{m+1}\right) \geq \operatorname{ind}\left(c^{m}\right), m \geq 1,
$$

cf. the successive index estimates by Long \& Zhu, cf. [20, [19, Sec. 10.2]. If $\operatorname{ind}\left(c^{2}\right) \equiv \operatorname{ind}(c)+1(\bmod 2)$ it follows from Inequality (8) and Equality (5) that

$$
\operatorname{ind}\left(c^{m+1}\right) \geq \operatorname{ind}\left(c^{m}\right)+1
$$

for all $m \geq 1$.

Now we discuss four cases depending on the parity of the dimension $n$ and the parity of ind $\left(c^{2}\right)-\operatorname{ind}(c)$.

Case 1: Assume $n$ even, then

$$
b_{k}= \begin{cases}2 ; & k=(2 j+1)(n-1), j \geq 1 \\ 1 ; & k \geq n-1, k \text { odd }, k \neq(2 j+1)(n-1), j \geq 1, \\ 0 \quad ; \quad \text { otherwise }\end{cases}
$$

cf. [22, Thm.2.4] resp. [15, p.104].

CASE 1.1: Assume in addition that $\operatorname{ind}\left(c^{2}\right) \equiv \operatorname{ind}(c)(\bmod 2)$, i.e. $\operatorname{ind}\left(c^{m}\right) \equiv$ $n-1(\bmod 2)$ for all $m \geq 1$. Then Equations (6), (7) and (8) imply for $k \leq N_{1}$ :

$$
w_{k}=\#\left\{m \in \mathbb{N} ; \operatorname{ind}\left(c^{m}\right)=k\right\}=b_{k},
$$

and

$$
\begin{array}{r}
\left(\operatorname{ind}\left(c^{m}\right)\right)_{m \leq m_{1}}=(n-1, n+1, \ldots, 3 n-5,3 n-3,3 n-3,3 n-1, \ldots, \\
\ldots, 5 n-7,5 n-5,5 n-5,5 n-3, \ldots) .
\end{array}
$$

In particular we obtain for all $m \leq m_{1}$ :

$$
\operatorname{ind}\left(c^{n m}\right)=(2 m+1)(n-1) \text {. }
$$

Since ind $\left(c^{2 n}\right)=5 n-5<N_{1}$ we obtain $m_{1} \geq 2$. And Equation (11) implies

$$
\operatorname{ind}\left(c^{n m}\right)-m \operatorname{ind}\left(c^{n}\right)=-(m-1)(n-1)
$$

for all $m \leq m_{1}$, hence Equation (11) holds for $r=n$.

CASE 1.2: Assume in addition that $\operatorname{ind}\left(c^{2}\right) \equiv \operatorname{ind}(c)+1(\bmod 2)$, i.e. $\operatorname{ind}\left(c^{m}\right) \equiv m(\bmod 2)$. Then Equations (6) $)$ (7) and (8) imply for $k \leq N_{1}$ :

$$
w_{k}=\#\left\{l \in \mathbb{N} ; \operatorname{ind}\left(c^{2 l-1}\right)=k\right\}=b_{k},
$$

and $\operatorname{ind}\left(c^{2 n-1}\right)=\operatorname{ind}\left(c^{2 n+1}\right)=3 n-3$ contradicting Inequality (9). Therefore this case cannot occur. 
CAse 2: Assume $n$ odd, then

$$
b_{k}= \begin{cases}2 & ; \quad k=j(n-1), j \geq 2 \\ 1 & ; k \geq n-1, k \text { even }, k \neq j(n-1), j \geq 2 \\ 0 & ; \quad \text { otherwise }\end{cases}
$$

cf. [22, Thm.2.4] resp. [15, p.104].

CAse 2.1: Aussume in addition that $\operatorname{ind}\left(c^{2}\right) \equiv \operatorname{ind}(c)(\bmod 2)$,

i.e. $\operatorname{ind}\left(c^{m}\right) \equiv 0(\bmod 2)$ for all $m \geq 1$. Then Equations (6), (7) and (8) imply for $k \leq N_{1}$ :

$$
w_{k}=\#\left\{l \in \mathbb{N} ; \operatorname{ind}\left(c^{l}\right)=k\right\}=b_{k},
$$

i.e.

$$
\begin{array}{r}
\left(\operatorname{ind}\left(c^{m}\right)\right)_{m \leq m_{1}}=(n-1, n+1, \ldots, 2 n-4,2 n-2,2 n-2,2 n, \ldots . \\
\ldots, 3 n-5,3 n-3,3 n-3,3 n-1, \ldots) .
\end{array}
$$

In particular we obtain for all $m \leq m_{1}$

$$
\operatorname{ind}\left(c^{m(n+1) / 2}\right)=(m+1)(n-1) \text {. }
$$

Since $\operatorname{ind}\left(c^{2(n+1)}\right)=5 n-5$ we obtain $m_{1} \geq 4$. Equation (13) implies

$$
\operatorname{ind}\left(c^{m(n+1) / 2}\right)-m \operatorname{ind}\left(c^{(n+1) / 2}\right)=-(m-1)(n-1)
$$

for all $m \leq m_{1}$, which is Equation (1) for $r=(n+1) / 2$.

CASE 2.2: Assume in addition that $\operatorname{ind}\left(c^{2}\right) \equiv n$ i.e. $\operatorname{ind}\left(c^{m}\right) \equiv m$ $(\bmod 2)$. Then Equations (6), (7) and (8) imply for $k \leq N_{1}$ :

$$
w_{k}=\#\left\{l \in \mathbb{N} ; \operatorname{ind}\left(c^{2 l-1}\right)=k\right\}=b_{k},
$$

and $\operatorname{ind}\left(c^{n}\right)=\operatorname{ind}\left(c^{n+2}\right)=2 n-2$ contradicting Inequality (9). Therefore this case cannot occur.

In Case 1.1 and Case $2.1 m_{1} \geq 2$ and $\operatorname{ind}\left(c^{2 r}\right)-2 \operatorname{ind}\left(c^{r}\right)=-(n-1)$. Therefore it follows from [2, Lemma 3.1(ii)] that $c$ is of elliptic-parabolic type. And the linearized Poincaré mapping decomposes into $(n-1)(2 \times 2)$ blocks conjugate to rotations.

\section{EXAMPLE}

For $\epsilon>0, \eta>0$ with $\eta+\epsilon<1 / 2$ let $h=h_{\epsilon, \eta}: \mathbb{R} \longrightarrow[0,1]$ be a smooth funtion satisfying

$$
h_{\epsilon, \eta}(t)=\left\{\begin{array}{llc}
1 & ; \quad t \in[-\eta, \eta] \\
0 & ; \quad|t| \geq \eta+\epsilon / 4
\end{array}\right.
$$

Let $H_{\epsilon}: \mathbb{R} \longrightarrow \mathbb{R}$ be the smooth function with $H_{\epsilon}(t)=\epsilon h_{\epsilon, \epsilon / 4}(t)$. And let $a=a_{\epsilon, \eta}: \mathbb{R} \longrightarrow[-1,1]$ be the smooth function with $a_{\epsilon, \eta}(t+2)=-a_{\epsilon, \eta}(t)$ and $a_{\epsilon, \eta}(1+t)=h_{\epsilon, \eta}(t)$ for all $-1 \leq t \leq 1$.

Let $p_{1}, p_{2}, \ldots, p_{n}$ be numbers which are relatively prime and let $p=$ $p_{1} p_{2} \cdots p_{n}$. Let $e_{0}, e_{1}, e_{2}, \ldots, e_{2 n}$ be an oriented orthonormal basis of $\mathbb{R}^{2 n+1}$. 
For the standard Riemannian metric on $S^{2 n} \subset \mathbb{R}^{2 n+1}$ an isometric $S^{1}$ action $\phi: S^{2 n} \times S^{1} \longrightarrow S^{2 n}$ is defined by $\phi\left(e_{0}, t\right)=e_{0}$ and

$$
\begin{aligned}
\phi\left(e_{2 j-1}, t\right) & =\cos \left(2 \pi p t / p_{j}\right) e_{2 j-1}+\sin \left(2 \pi p t / p_{j}\right) e_{2 j} \\
\phi\left(e_{2 j}, t\right) & =-\sin \left(2 \pi p t / p_{j}\right) e_{2 j-1}+\cos \left(2 \pi p t / p_{j}\right) e_{2 j} .
\end{aligned}
$$

with $j=1,2, \ldots, n$. We assume that there is a non-reversible Finsler metric $f$ invariant under $\phi$ for which there are only finitely many geometrically distinct closed geodesics $c_{1}, c_{1}^{-1}, c_{2}, c_{2}^{-1}, \ldots, c_{n}, c_{n}^{-1}$ which occur in pairs differing only by orientation.

The geodesic $c_{j}, j=1,2, \ldots, n$ is the intersection of the 2-plane generated by $e_{2 j-1}, e_{2 j}$ invariant under $\phi$, i.e. $c_{j}(t)=\cos (2 \pi t) e_{2 j-1}+\sin (2 \pi t) e_{2 j}$. The closed geodesics $c_{j}, j=1,2, \ldots, n$ do not intersect pairwise.

This assumptions are satisfied by the Katok metrics $N_{\alpha}$ on $S^{2 n}$ with irrational $\alpha \in(0,1)$ discussed in detail by Ziller [26, Sec.1]. Here the lengths $L\left(c_{j}\right), L\left(c_{j}^{-1}\right)$ of the closed geodesics $c_{j}, c_{j}^{-1}$ depend on the parameter $\alpha$.

Proposition 3. Let $f$ be a bumpy Katok metric on $S^{2 n}$ invariant under the $S^{1}$-action $\phi$ by Equation (14) with only $2 n$ geometrically distinct closed geodesics $c_{1}, c_{1}^{-1}, \ldots, c_{n}, c_{n}^{-1}$ and let $L_{1}:=\max \left\{L\left(c_{j}\right), L\left(c_{j}^{-1}\right), j=\right.$ $1,2, \ldots, n\}$. The $n$ closed geodesics $c_{1}, \ldots, c_{n}$ are invariant under $\phi$ and do not intersect pairwise.

Fix $k \geq 4$. For any $L>L_{1}$ there is a non-reversible Finsler metric $F$ invariant under $\phi$ of positive flag curvature and $C^{k}$-arbitrarily close to $f$ with only $2 n$ geometrically distinct closed geodesics $d_{1}, d_{2}, \ldots, d_{2 n}$ of length $<L$. These $2 n$ closed geodesics are invariant under $\phi$ and do not intersect pairwise.

Fix the closed geodesic $c=c_{1}$ and $m=m_{1}=p / p_{1}$. Then there is a $S^{1}$-invariant tubular neighborhood $U(c)$ of the closed geodesic $c_{1}$ with coordinates

$$
(x, t) \in D_{\epsilon}^{2 n-1} \times_{\mathbb{Z}_{m}} S^{1} \longmapsto \phi\left(\cos (\|x\|) e_{1}+\sin (\|x\|) \frac{x}{\|x\|}, t\right) \in U(c)
$$

Here $D_{\epsilon}^{2 n-1}=\left\{x \in \mathbb{R}^{2 n-1} ;\|x\|<\epsilon\right\}$ is a disc of radius $\epsilon$ on the Euclidean space generated by $e_{0}, e_{3}, e_{4}, \ldots, e_{2 n}$ which we identify via the exponential map of the standard Riemannian metric on $S^{2 n}$ with an open neigborhood of $c(0)=e_{1}$ on the totally geodesic hypersphere through $c(0)$ orthogonal to the $S^{1}$-action.

The $S^{1}$-action induces an isometric $\mathbb{Z}_{m}$-action on $D_{\epsilon}^{2 n-1}$, and therefore a diagonal action on the product $D_{\epsilon}^{2 n-1} \times S^{1}$ with quotient space $D_{\epsilon}^{2 n-1} \times_{\mathbb{Z}_{m}} S^{1}$. Then there are induced coordinates $(x, t, \xi, \tau) \in\left(D_{\epsilon}^{2 n-1} \times_{\mathbb{Z}_{m}} S^{1}\right) \times \mathbb{R}^{2 n-1} \times \mathbb{R}$ on the tangent bundle $T U(c)$ restricted to the tubular neighborhood $U(c)$ of $c$. Because of the $S^{1}$-symmetry the Finsler metric $f$ in the coordinates $(x, t, \xi, \tau)$ does not depend on $t$, i.e. $f(x, t, \xi, \tau)=f(x, \xi, \tau)$.

Choose $\eta \in(0,1 / 4)$ and a sufficiently small $\epsilon>0$. For a sufficiently small $s>0$ the mapping $\Psi=\Psi_{s, \epsilon, \eta}: T U(c)-T^{0} U(c) \longrightarrow T U(c)-T^{0} U(c)$ of the 
tangent bundle $T U(c)$ of the tubular neighborhood minus the zero section $T^{0} U(c)$ defined by

$$
\Psi(x, t, \xi, \tau)=\left(x+s H_{\epsilon}(\|x\|) a_{\eta, \epsilon}\left(\frac{\tau}{\sqrt{\tau^{2}+\|\xi\|^{2}}}\right) e_{0}, t, \xi, \tau\right)
$$

is a diffeomorphism. For a fixed $k \geq 4$ one can choose $s>0$ sufficiently small such that the diffeomorphism $\Psi_{s, \epsilon, \eta}$ is arbitrarily close to the identity map in the $C^{k}$-norm. The mapping is well-defined since $e_{0}$ is fixed under $\phi$.

The diffeomorphism $\Psi$ extends by the identity onto the complement of $T U(c)$ on $T S^{2 n}$. And the diffeomorphism $\Psi$ is positively homogeneous of degree 1, i.e. $\Psi(x, t, \lambda \xi, \lambda \tau)=\lambda \Psi(x, t, \xi, \tau)$ for $\lambda>0$.

Then define the Finsler metric

$$
\bar{f}(x, t, \xi, \tau)=\bar{f}_{s, \epsilon, \eta}(x, t, \xi, \tau)=f\left(\Psi_{s, \epsilon, \eta}^{-1}(x, t, \xi, \tau)\right) .
$$

Let $V_{ \pm}=\left\{(x, t, \xi, \tau) ;\|x\|<\epsilon / 4, \pm \tau / \sqrt{\tau^{2}+\|\xi\|^{2}}>1-\eta\right\}$ and $V_{1}=\left\{(x, t, \xi, \tau) ;|\tau| / \sqrt{\tau^{2}+\|\xi\|^{2}} \leq 1-\eta-\epsilon / 4\right\}$. Then

$$
\bar{f}_{s, \epsilon, \eta}(x, t, \xi, \tau)=\left\{\begin{array}{cl}
f(x, t, \xi, \tau) & ; \quad(x, t, \xi, \tau) \in V_{1} \\
f\left(x \mp s \epsilon e_{0}, t, \xi, \tau\right) & ; \quad(x, t, \xi, \tau) \in V_{ \pm}
\end{array} .\right.
$$

Therefore we obtain that $d_{1}: t \mapsto\left(s \epsilon e_{0}, t\right)$ and $d_{2}: t \mapsto\left(-s \epsilon e_{0},-t\right)$ are two closed geodesics of $\bar{f}_{s, \epsilon, \eta}$ in $U(c)$ which do not intersect.

The flag curvature of the Katok metric is constant with value 1, cf. [23, Sec.4]. And we obtain that the flag curvature $\bar{K}(y, Y, \sigma)$ of a flag $(y, Y, \sigma)$ with $y \in S^{2 n}, Y \in T_{y} S^{2 n}$ and $\sigma$ a two-dimensional subspace of $T_{y} S^{2 n}$ containing $y$ with respect to the perturbed Finsler metric $\bar{f}=\bar{f}_{s, \eta, \epsilon}$ can be different from 1 only if $y \in U(c)$ and if the coordinates $(x, t, \xi, \tau)$ of the tangent vector $Y$ satisfy: $(x, t, \xi, \tau) \notin V_{1} \cup V_{+} \cup V_{-}$. The Finsler metric $\bar{f}_{s, \epsilon, \eta}$ is invariant under $\phi$. But the symmetry with respect to the reflection at the $\left(e_{1}, e_{2}\right)$-plane is broken.

We can use this perturbation inductively in tubular neighborhoods for all closed geodesics $c_{2}, \ldots, c_{n}$ and obtain a non-reversible Finsler metric $F=F_{s, \epsilon, \eta}$ on $S^{2 n}$ invariant under $\phi$ with closed geodesics $d_{3}, d_{4}, \ldots, d_{2 n}$ also invariant under $\phi$. Any two of these closed geodesics do not intersect.

Now fix $L>L_{1}$. Since $f$ is bumpy there is a sufficiently small $s>0$ such that all closed geodesics of the Finsler metric $F_{s, \epsilon, \eta}$ of length $<L$ are geometrically equivalent to $d_{1}, d_{2}, \ldots, d_{2 n}$, for this argument compare for example [1, p.12, 13]. Here we use the strong $C^{k}$-topology with $k \geq 4$ (instead of $k \geq 2$ in the Riemannian case) since in contrast to the Riemannian case the geodesic coefficients depend on fourth derivatives of the Finsler metric.

Since by Equation (15) the Finsler metrics $f$ and $F=F_{s, \epsilon, \eta}$ are isometric in open neighbhorhoods of the velocity fields $c_{j}^{\prime}$ and $d_{2 j-1}^{\prime}$ resp. $-c_{j}^{\prime}$ and $d_{2 j}^{\prime}$ for $j=1,2, \ldots, n$ the flag curvature is constant in these neighborhoods and the lengths $L\left(c_{j}\right)=L\left(d_{2 j-1}\right), L\left(c_{j}^{-1}\right)=L\left(d_{2 j}\right), j=1,2, \ldots, n$ coincide. 
Therefore the picture from Morse theory of $f$ produced by $c_{1}, c_{1}^{-1}, \ldots, c_{n}, c_{n}^{-1}$ and $F$ produced by $d_{1}, d_{2}, \ldots, c_{2 n}$ up to length $L$ coincide.

For $n=2$ it follows from [14, Sec.6] that also the perturbed metric $F$ on $S^{2}$ is dynamically convex. Therefore there are either infinitely many geometrically distinct closed geodesics or there are only two geometrically distinct closed geodesics.

For $n=2$ we obtain non-reversible and rotationally invariant Finsler metrics in any neighborhood of the Katok metric with two closed geodesics $d_{1}, d_{2}$ also invariant under the $S^{1}$-action which do not intersect. For existence results for closed geodesics invariant under isometries cf. 24, Prop.2, Prop.3].

\section{REFERENCES}

[1] D.V.Anosov: On generic properties of closed geodesics (Russian) Izv.Akad.Nauk. SSSR 46 (1982) = (Engl. transl.) Math. USSR Izv. 21 (1983) $1-29$

[2] W.Ballmann, G.Thorbergsson \& W.Ziller: Closed geodesics on positively curved manifolds. Ann. Math. (2) 116 (1982) 213-247

[3] V.BAngert \& Y.Long: The existence of two closed geodesics on every Finsler 2-sphere. Math. Ann. 346 (2010) 335-366

[4] G.D.Birkhoff: Dynamical systems with two degrees of freedom. Trans. Amer. Math. Soc. 18 (1917) 199-300

[5] G.D.Birkhoff: Dynamical Systems. Amer. Math. Soc. Coll.Publ. Vol. IX, Amer.Math.Soc. New York 1927

[6] R. Bотт: On the iteration of closed geodesics and the Sturm intersection theory, Comm. Pure Appl. Math 9 (1956) 171-206

[7] H.DuAn \& Y.LONG: Multiple closed geodesics on bumpy Finsler spheres. J.Diff.Eq. 233 (2007) 221-240

[8] H.Duan \& Y.Long: Multiple closed geodesics on 3-spheres. Adv. Math. 221 (2009) 1757-1803

[9] H.DuAn \& Y.LOnG: The index growth and multiplicity of closed geodesics, J. Funct. Anal. 259 (2010) 1850-1913

[10] H.DuAn \& Y.Long: The index quasi-periodicity and multiplicity of closed geodesics. arXiv:1008.1458

[11] H.DuAn, Y.Long \& W.WANG: The enhanced common index jump theorem for symplectic paths and non-hyperbolic closed geodesics on Finsler manifolds, arXiv:1510.02872

[12] A.I.Fet: A periodic problem in the calculus of variations. Dokl. Akad. Nauk SSSR (N.S.) 160 (1965) 287-289 (russ.)= Sov.Mathem. 6 (1965) 85-88

[13] M.Goresky \& N.Hingston: Loop products and closed geodesics, Duke Math.J. 150 (2009) 117-209

[14] A.HARRis \& G.PATERnAin: Dynamically convex Finsler metrics and J-holomorphic embedding of asymptotic cylinders, Ann. Global Anal. Geom. 34 (2008)115134

[15] N.Hingston: Equivariant Morse theory and closed geodesics. J.Differential Geom. 19 (1984) 85-116

[16] N. Hingston: On the growth of the number of closed geodesics on the two-sphere, Intern. Math. Res. Notices 9 (1993) 253-261

[17] N.Hingston \& H.B.RademacheR: Resonance for loop homology of spheres, J. Differential Geom. 93 (2013) 133-174 
[18] A.B.KATOK: Ergodic pertubations of degenerate integrable Hamiltonian systems. Izv.Akad.Nauk SSSR Ser.Mat. 37(1973) 539-576 (Russian)= (Engl.translation) Math. USSR Izv.7 (1973) 535-572

[19] Y.LonG: Index theory for symplectic paths with applications, Progress Math. 207, Birkäuser, Basel, Boston, Berlin 2002

[20] Y.Long \& C.ZHU: Closed characteristics on compact convex hypersurfaces in $\mathbb{R}^{2 n}$, Ann.Math. (2) 155 (2002) 317-368

[21] L.A. LyUsteRnik \& A.I.Fet: Variational problems on closed manifolds. (Russian) Dokl. Akad. Nauk SSSR (N.S.) 81 (1951) 17-18

[22] H.B.RADEmacher: On the average indices of closed geodesics. J.Differential Geom. 29 (1989) 65-83

[23] H.B.Rademacher, A sphere theorem for non-reversible Finsler metrics. Math. Ann. 328 (2004) 373-387

[24] H.B.RADEMACHER, Existence of closed geodesics on positively curved Finsler manifolds. Erg. Th. \& Dyn. Syst. 27 (2007) 251-260

[25] H.B.RADEMACHER: The second closed geodesic on Finsler spheres of dimension $n>2$, Trans. American Math. Soc. 362 (2010) 1413-1421

[26] W.Ziller: Geometry of the Katok examples. Ergod.Th.\& Dyn.Syst. 3 (1982) $135-157$

Mathematisches Institut, Universität Leipzig, 04081 Leipzig, Germany

E-mail address: rademacher@math.uni-leipzig.de 